Applied Remote Sensing

RemoteSensing.SPIEDigitalLibrary.org

\title{
2018 List of Reviewers
}

SPIE. “2018 List of Reviewers,” J. Appl. Remote Sens. 13(1), 010101 (2019), 
The Journal of Applied Remote Sensing would like to sincerely thank the following individuals who served as reviewers in 2018. The success of our publication hinges on the voluntary contributions of time and energy put forth by these professionals.

\begin{tabular}{|c|c|}
\hline Mohammed Abbas Kadhim & Jeremy Bos \\
\hline Elfatih Abdel-Rahman & Emmanuel Boss \\
\hline Saygin Abdikan & Salah Bourennane \\
\hline Abhijat Abhyankar & Michael Bowlan \\
\hline Nicola Acito & Brian Brisco \\
\hline Mariana Adam & Henning Buddenbaum \\
\hline Paolo Addesso & Krishna Buddhiraju \\
\hline Gabriela Afrasinei & Merv Budge \\
\hline Meghna Agarwala & Andrea Buono \\
\hline Azita Ahmad Zawawi & Arturo Cadena \\
\hline Mozhgan Ahmadi Nadoushan & Marcello Caleffi \\
\hline Chi Ahn & Fulvio Capodici \\
\hline Jiaqiu Ai & Toby Carlson \\
\hline Hayder Al-Salihi & Davide Cataldo \\
\hline Afshar Alam & Jack Cederquist \\
\hline Abdulraqeb Alhammadi & Catherine Champagne \\
\hline Imama Ali & Chien-Ping Chang \\
\hline Umit Alkus & Jose Chavez \\
\hline Jesus Alvarez-Mozos & Pavel Cheben \\
\hline Clark Amerault & Bassam Chehade \\
\hline Daoxiang An & Bo Chen \\
\hline Anupam Anand & Chen Chen \\
\hline Jasdeep Anand & Hongmeng Chen \\
\hline Eric Anderson & Lin Chen \\
\hline Albert Ansmann & Shaohui Chen \\
\hline Erchan Aptoula & Zhikang (Ken) Chen \\
\hline Dildora Aralova & Chih-Yung Cheng \\
\hline Francisco Arguello & Gong Cheng \\
\hline Fernando Arias & Jerzy Chmiel \\
\hline Abd. Rahman Assyakur & Julian Christou \\
\hline Dogan Aydal & Rok Ciglic \\
\hline Rodolfo B. Arango & Davide Colombo \\
\hline James Baer & Christopher Crawford \\
\hline Xueru Bai & Giovanni Crosta \\
\hline Shahbaz Baig & Antonio D'Andrea \\
\hline John Ball & Guido D’Urso \\
\hline A. Banu & Farzaneh DadrasJavan \\
\hline Rory Barton-Grimley & Matthew Dannenberg \\
\hline Sukanta Basu & Shaunak De \\
\hline Tyas Basuki & Jorge de la Calleja \\
\hline Alireza Behrad & Cleon Dean \\
\hline Amin Beiranvand Pour & Wu Deng \\
\hline Mikhail Belenkii & Nuria Devanthery \\
\hline Mariana Belgiu & Anindita Dey \\
\hline V. A. Belokurov & Hayder Dibs \\
\hline Katja Berger & Jules Dim \\
\hline Segio Bernabe & Elias Dimitriou \\
\hline Bhashyam Bhashyam & David Diner \\
\hline Jignesh Bhatt & Baiyuan Ding \\
\hline Hamidullah Binol & Denis Dion \\
\hline Ksenia Bittner & Mathias Disney \\
\hline Marcelo Biudes & David Doelling \\
\hline Hussein Bizimana & Eric Donley \\
\hline Andrew Bodkin & Jack Dostalek \\
\hline Lionel Bombrun & JianFang Dou \\
\hline Boris Bonn & Jinyang Du \\
\hline Mitchell Bonney & Yannick Duguay \\
\hline Anatoli Borovoi & Ilya Dzyuban \\
\hline
\end{tabular}

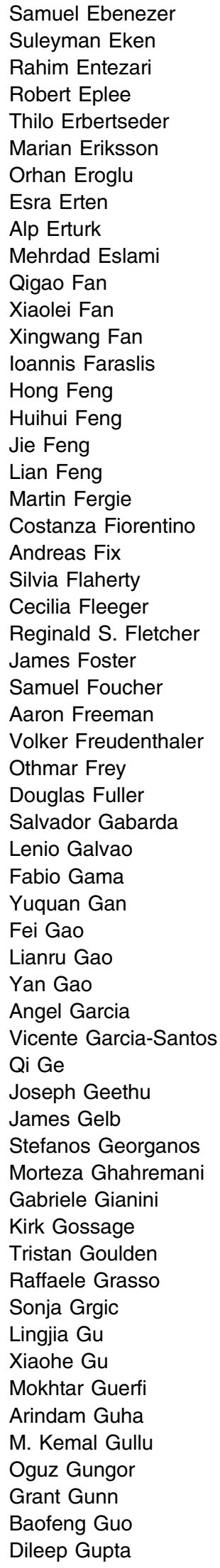


Sharad Gupta

Ali Gurbuz

Ofer Hadar

Ahmed Hagag

Nathan Hagen

Alireza Hamedianfar

Jungong Han

Wei Han

Michael Hart

Seyed Armin Hashemi

Mohammed Imamul Hassan Bhuiyan

Jennifer Hawley

Khaled Hazaymeh

Bin $\mathrm{He}$

Chuangjiang $\mathrm{He}$

Lei $\mathrm{He}$

Lin $\mathrm{He}$

Xingyu $\mathrm{He}$

Mary Henry

Stephen Herbst

Ittai Herrmann

Eric Hetland

Benjamin Heumann

Andrew Heymsfield

Paul Higgins

Donald Hillger

Jonathan Holland

Richard Holmes

Mauro Holzman

Saeid Homayouni

Paul Honeine

Masahiro Hori

Wen Hua

Fang Huang

Yan Huang

Eric Hudier

David Hulslander

Juliane Huth

Edurne Ibarrola-Ulzurrun

Amber ller

Markus Immitzer

Seema Jalan

Ali Akbar Jamali

Akhtar Jamil

Nasehe Jamshidpour

Yuhe Ji

Binbin Jiang

Junjun Jiang

Ruinian Jiang

Wen Jiang

Zhangyan Jiang

Xiaomeng Jin

Xiaoying Jin

Xin Jin

Linhai Jing

Robert Johnson

Mohamad Jouni

Dieter Just

Vipin Kamble

Xudong Kang
Nur Huseyin Kaplan

Mudit Kapoor

Ali Can Karaca

Vassilia Karathanassi

Ram Karsh

Ingebjorg Kasen

Z. M. Kassa

Jason Kaufman

Taskin Kavzoglu

Philippe Keckhut

Norman Kerle

Ramesh Kestur

Saeed Khabbazan

Joe Khalife

Muhammad Jaleed Khan

Unmesh Khati

Dennis Killinger

Keith Knox

Dilek Koc-San

Upama Koju

Levente Kovacs

Robert Kuligowski

Anil Kumar

Shashi Kumar

Vineet Kumar

Deepak Kumar Jain

Wattanapong Kurdthongmee

Mehmet Kurum

Tiit Kutser

Christos Kyrkou

Salim Lamine

Charis Lanaras

Amir Laribi

Andon Lazarov

Thu Trang Le

Vannhu Le

Ellsworth LeDrew

Moritz Lennert

Kevin Leonard

Dietmar Letalick

David W. Leverington

Eugene Levin

Ainong Li

Bo Li

Guicai Li

Heng-Chao Li

Hengchao Li

Hui Li

Jia Li

Jiang Li

Jianwei Li

Jun Li

Jun Li

Peijun Li

Shanshan Li

Xi Li

Xiangli Li

Xinghua Li

Xingong Li

Xueyuan Li
Yansheng Li

Yanyan Li

Yundong Li

Zengyuan Li

Miaomiao Liang

Qilian Liang

Shouzhen Liang

Veraldo Liesenberg

Caiyong Lin

Wenpeng Lin

Truong-Hong Linh

Jorge Lira

Bing Liu

Bo Liu

Chian-Yi Liu

Daijun Liu

Hongying liu

Huiying Liu

Jianhong Liu

Jiqiao Liu

Kai Liu

Leilei Liu

Meiling Liu

Qiegen Liu

Shiliang Liu

Shuaiqi Liu

Sicong Liu

Xiangnan Liu

Xiaoye Liu

Yi Liu

Yuanbo Liu

Zhandong Liu

Zhengjun Liu

Bing Lu

Vladimir Lukin

Xiaoyan Luo

Alberto Lupidi

Aidy M Muslim

Chao Ma

Lei Ma

Li Ma

Ronghua Ma

Mehdi Maboudi

Celia Machado

Bomidi Madhavan

Konstantinos Makantasis

Constantino Malagon

Antonino Maltese

Dimitris Manolakis

Chongyuan Mao

Feiyue Mao

Javier Marcello-Ruiz

Maged Marghany

Daniele Marinelli

Armando Marino

Jose Marino

Andrea Marinoni

Isabella Mariotto

Brian Markham

Tapas Martha 
David Mason

Antoine Masse

Vidhya Mathivanan

Stefania Matteoli

Himanshu Maurya

Ronald McRoberts

Sivasubramanyam Medasani

Mustapha Meftah

Anand Mehta

Mohamed Lamine Mekhalfi

Jihua Meng

Erzsebet Merenyi

Ibrahim Mesecan

Pu Miao

Qiguang Miao

Suresh Middinti

Jarno Mielikainen

Katarzyna Mirek

Ioannis Mitsopoulos

Tomoaki Miura

Mohammad Reza Mobasheri

Christopher Moeller

Alkhatib Mohammed

Fernando Moreira de Araujo

Ana Moreira Teodoro

Abdulmajid A. Mrebit

Farrah Melissa Muharam

Saumitra Mukherjee

Justin Murfitt

Kalaiyarasi Murugesan

Massimo Musacchio

Andreas Muschinski

Abdullah Nahid

Venkata Narra

Venkatanathan Natarajan

Biswajit Nath

Rodrigo Nava

Gabriel Navarro

Majid Nazeer

Amro Negm

Rogerio Negri

Brian Ng

Sheng Nie

Jifeng Ning

Victor Nosov

Sean O'Brien

Jarlath O'Neil-Dunne

Kenta Obata

Akpona Okujeni

Colin Olson

Isin Onur

Thomas Oommen

Irena Orovic

Ahmad Osman

Mohammed Ouali

Asli Ozdarici-Ok

Todd Paciencia

N. Padmanabhan

Mary Pagnutti

Riccardo Palama
Shailesh Panchal

Mercedes E. Paoletti

Matteo Pardini

Eberhard Parlow

Edoardo Pasolli

Jigisha Patel

C. Patnaik

Zdenek Patocka

Swarnajyoti Patra

Anand Paul

Audrey Paulus

Kabir Peerbhay

Jifang Pei

Jian Peng

Zhenming Peng

Antonio Pepe

Richard Pfisterer

Hai Pham

Mark Phillips

Marcelo Pinho

Nicole Pinnel

Claudia Pipitone

Joao Pompeu Pavanelli

Mangalraj Poobalasubramanian

Nitesh Poona

Mohammed Reza Poormir

Guy Potvin

Kusuma Prabhakara

Manoharan Prabukumar

Ganesh Prasad

Saurabh Prasad

G. Prates

Thomas Puestow

Jeffrey Puschell

Lijuan Qi

Lilong Qin

Xianlin Qin

Chunyan Qu

Lindi Quackenbush

Miguel Quemada

Farhad Rad

Mahmudur Rahman

Upendra Rajput

Shanmuganathan Raman

Vinod Ramnath

Abel Ramoelo

Raaj Ramsankaran

Cuddapah Rao

Asma Rasheid Shora

Mehul Raval

Diego Reale

Asid ur Rehman

Guoquan Ren

William Reynolds

Martina Ricko

Nicolina Ripa

Haris Riris

Gianfranco Rizzo

Dar Roberts

Komeil Rokni
Domenico Ronga

Neil Rowlands

Ana Ruiz-Constan

Filip Sabo

Dario Sacco

Sudipan Saha

Santo Salinas

Siti Aekbal Salleh

Vincent Salomonson

Carl Salvaggio

Federica Salvetti

Alim Samat

Sarah Hanim Samsudin

Yukihisa Sanada

Juan M. Sanchez

Teresa Santos

Asiya Sarinova

Firas Sawaf

Michael Sayers

Randall Scharien

Stephanie Schieffer

Timothy Schmit

Amanda M. Schwantes

Imane Sebari

Umut Sefercik

Alfonso Senatore

Jeong Seong

Mahdi Shabankareh

Reza Shah-Hosseini

Mozhdeh Shahbazi

Morteza Shahriari Nia

Amit Shakya

Hui Shao

Xi Shao

Xiaopeng Shao

Zhenfeng Shao

J.B. Sharma

Chaomin Shen

Hongyin Shi

Zhenwei Shi

Manoochehr Shirzaei

Greg Showman

Mbulisi Sibanda

Muhammad Adnan Siddique

Pratap Singh

Sudhir Singh

Avik Sinha

V. Sivakumar

Ramesh Sivanpillai

Lars Sjoqvist

Sergii Skakun

Karl Skretting

Terrence Slonecker

Richard Smith

Hilary E. (Ned) Snell

Peter Somkuti

Nguyen-Thanh Son

Kaishan Song

Foo Chong Soon

Marios Spiliotopoulos 
Hari Shanker Srivastava

Demetris Stathakis

Joshua Stewart

Tom Stone

Hongbo Su

Hongjun Su

Jia Su

Yuanchao Su

Haigang Sui

Genyun Sun

Sheng Sun

Xiaoli Sun

Zhibin Sun

Christopher Sundar

Olga Sykioti

James Szykman

Muhammad Naveed Tahir

Ke Tan

Kun Tan

Yumin Tan

Guiqian Tang

Hao Tang

Van Ha Tang

Yuchao Tang

Alireza Taravat

Gulsen Taskin

Martin Tauc

Khaoula Tbarki

Sergio Teggi

Peter J.G. Teunissen

Prasad Thenkabail

Pierre-Antoine Thouvenin

R. Thriveni

Feng Tian

Qingjiu Tian

Zhiqiang Tian

Lei Tong

Melanie Trudel

Philemon Tsele

Esra Tunc Gormus

Eija Tuominen

Andreas Tzanis

Tatsumi Uezato

Kishor Upla

Mustafa Ustuner

Faruk Uysal

Stephanie Uz

Andrea Vaccari

Radhika Vadhi

Preethi Vaidyanathan

Ruben Valbuena

Diego Valsesia

Piet van Genderen

Miguel Velez-Reyes

Marie Therese Velluet

Jayaraj Velusamy

Dmitry Vengertsev
Marie-Leen Verdonck

Miguel Villarreal

Matias Viotti

Oskar von der Luhe

Christian von Savigny

Alaaddin Vural

Sebastien Wagner

Philippe Waldteufel

Xianrong Wan

Cuizhen Wang

Hongquan Wang

Jianping Wang

Ling Wang

Lizhe Wang

Panshi Wang

Qi Wang

Qunming Wang

Wen Wang

Xiaoyang Wang

Yinghua Wang

Yong Wang

Yuexian Wang

Zhangyang Wang

Zhaofa Wang

Zhuozheng Wang

Lianhuan Wei

Tian Wei

Aaron Weiskittel

Gezheng Wen

Jinhuan Wen

Jinming Wen

Qian Weng

Norman Wildmann

Kendall Wnuk

Aisheng Wu

Chaofan Wu

Chen Wu

JingWei Wu

Keyu Wu

Qiusheng Wu

Yonghua Wu

Yulian Wu

Shaoyan Xia

Fei Xing

Mengdao Xing

Jin Xu

Lili $X u$

Qizhi Xu

Xingjian Xu

Irene Xueref-Remy

Xing Yan

Chao Yang

Fanlin Yang

Qingsong Yang

Wankou Yang

Xiaomei Yang

Xiucheng Yang
Yingbao Yang

Zhijing Yang

Guobiao Yao

Bhavani Kumar Yellapragada

Volkan Yilmaz

Jiong You

Yanan You

Qiang Yu

Linwei Yue

Peter Yuen

Konstantin Yushkov

Yuhendra Yusuf

Tanish Zaveri

Alexander Zemlyanov

Chuangzhan Zeng

Bing Zhang

Chengyan Zhang

Chengye Zhang

Chuanrong Zhang

Fan Zhang

Guoqing Zhang

Hankui Zhang

Haopeng Zhang

Jiahua Zhang

Liangpei Zhang

Lifu Zhang

Qilei Zhang

Shaoquan Zhang

Wei Zhang

Xi Zhang

Yanbo Zhang

Yu Zhang

Yunhua Zhang

Chuanfeng Zhao

Huimin Zhao

Lingli Zhao

Ningning Zhao

Shuhe Zhao

Yanyu Zhao

Yindi Zhao

Haiyong Zheng

Zezhong Zheng

Mikhail Zhizhin

Liheng Zhong

Yanfei Zhong

Ji Zhou

Wenzuo Zhou

Xiaolu Zhou

Xiran Zhou

Bingcheng Zhu

Peng Zhu

Xiaolin Zhu

Andrea Zingoni

Roghayeh Zoleikani

Kiana Zolfaghari

Bin Zou

Mehrez Zribi 